\title{
Radiation trapping in coherent media
}

\author{
A. B. Matsko, I. Novikova, M. O. Scully, and G. R. Welch \\ Department of Physics, Texas AEM University, College Station, Texas 77843-4242,
}

(October 31, 2018)

\begin{abstract}
We show that the effective decay rate of Zeeman coherence, generated in a ${ }^{87} \mathrm{Rb}$ vapor by linearly polarized laser light, increases significantly with the atomic density. We explain this phenomenon as the result of radiation trapping. Our study shows that radiation trapping must be taken into account to fully understand many electromagnetically induced transparency experiments with optically thick media.
\end{abstract}

Typeset using REVTEX 
Coherent population trapping (CPT) was first observed in experiments establishing Zeeman coherence in sodium atoms [罒. In these experiments, explained in terms of a three-level $\Lambda$-type level scheme, a laser field was used to create superpositions of the ground state sublevels. One of these superpositions, referred to as the "bright" state, can interact with the laser field while the other superposition does not and is referred to as the "dark" state [2]. All the population in the system is eventually optically pumped into the dark state, and resonant absorption of the electromagnetic field almost disappears. This phenomenon is one manifestation of electromagnetically induced transparency (EIT) [2,[].

EIT is particularly interesting because it offers a wide variety of applications ranging from lasers without population inversion [4, 5] to new trends in nonlinear optics [6]. One of the most striking phenomena connected with EIT is that atoms prepared in a coherent superposition of states can produce a steep dispersion and a large resonant index of refraction with vanishing absorption [7]. Preparation of matter in such a state (which has been dubbed "phaseonium" [5]) provides us with a new type of optical material of interest both in its own right, and in many applications to fundamental and applied physics.

A common condition for applications of EIT is a high optical density of the resonant medium. For example, in experiments demonstrating enhancement of index of refraction $\left(\chi^{\prime} \sim 10^{-4}\right)$ the density of the particles was $N \sim 10^{12} \mathrm{~cm}^{-3}[7]$. Also, for highly sensitive magnetometry based on atomic phase coherence the density of atoms is estimated to be $N \sim 5 \times 10^{12} \mathrm{~cm}^{-3}[8$.

For optically thick media, reabsorption of spontaneously emitted photons can become important. This process, called radiation trapping, has been studied extensively in astrophysics, plasma physics, and atomic spectroscopy [9]. Radiation trapping has been predicted and demonstrated to have a destructive effect on the orientation produced by optical pumping $[10[12$. Because the spontaneously emitted photons are dephased and depolarized with respect to the coherent fields creating the atomic polarization, the effect of radiation trapping can be described as an external incoherent pumping of the atomic transitions [12]. Under the conditions of EIT, there are not many atoms undergoing spontaneous emission. How- 
ever, these spontaneous photons destroy the atomic coherence in the same way as incoherent pumping. This effect can change the results of CPT and EIT experiments significantly.

In this Letter we report the observation and analysis of the increase of the effective decay rate of Zeeman coherence due to radiation trapping. We create the coherence between ground state sublevels of the $\mathrm{D}_{1}$ line of ${ }^{87} \mathrm{Rb}$ vapor, and study the effect of $\mathrm{CPT}$ as a function of the vapor density using polarization spectroscopy based on nonlinear magneto-optic rotation (NMOR) 13 15. We see that the the relaxation time of the coherent state is determined not only by the time-of-flight of the atom through the laser beam, but also by the density of the atomic vapor. For atomic densities $N \approx 5 \times 10^{12} \mathrm{~cm}^{-3}$ the effective coherence decay rate increases by several times compared with the decay rate for $N \approx 5 \times 10^{11} \mathrm{~cm}^{-3}$. This demonstrates the importance of radiation trapping for experiments with optically thick coherent media, and the need to account for it to understand the experimental results.

Our experimental setup is shown schematically in Fig. 1. An external cavity diode laser is tuned in the vicinity of the $F=2 \rightarrow F^{\prime}=1$ transition of the ${ }^{87} \mathrm{Rb} \mathrm{D}_{1}$ line. The laser beam passes through a high-quality polarizer $P_{1}$, and then through a cylindrical glass cell containing isotopically enhanced ${ }^{87} \mathrm{Rb}$. The length of the cell is $L=5.0 \mathrm{~cm}$ and its and diameter is $D=2.5 \mathrm{~cm}$. The laser power after the polarizer is $P=2.5 \mathrm{~mW}$, and the beam diameter is $d=2 \mathrm{~mm}$. The glass cell is placed inside a two-layer magnetic shield to suppress the laboratory magnetic field. A homogeneous longitudinal magnetic field is created by a solenoid mounted inside the inner magnetic shield. The density of the Rb vapor is controlled with the temperature of the cell. A second polarizer $P_{2}$ (a polarizing beam splitter) is placed after the cell and is tilted at $45^{\circ}$ with respect to the first polarizer. The two beams emerging from the polarizing beam splitter are detected with detectors $D_{1}$ and $D_{2}$. A simple analysis of the signals from the two channels gives the angle of rotation of the polarization $\phi$ and the transmitted intensity $I_{\text {out }}$.

We consider the linearly polarized light as two circular components $E_{+}$and $E_{-}$which generate a coherent superposition of the Zeeman sublevels (a dark state). To study this dark state we apply a longitudinal magnetic field in the direction of light propagation which 
leads to a splitting of the $\left|b_{ \pm}\right\rangle$states of magnitude $\hbar \delta_{0} / 2=-\mu_{B} B$ where $B$ is the magnetic field, $\mu_{B}$ is the Bohr magneton. Because the two circular components interact with ground-state sublevels of oppositely signed magnetic quantum number, the changes in the index of refraction for the two components have opposite sign. As a result of the index change, the components acquire a relative phase shift $\phi$ which leads to a rotation of the polarization direction which is proportional to the magnetic field for small fields. Thus, by measuring the rotation as a function of applied magnetic field, we obtain information about the dispersive properties of the medium. As the magnetic field increases, the absorption also increases because the splitting between the sublevels destroys the two-photon resonance, so the detection of the transmitted electromagnetic field intensity as a function of the magnetic field allows us to study the absorptive properties of the medium. Hence, using the nonlinear Faraday technique we can easily study both dispersive and absorptive properties of the EIT resonance simultaneously.

We have measured the polarization rotation slope $d \phi / d B$ and the transmission $I_{\text {out }} / I_{\text {in }}$ of the cell. The detailed shape depends on the particular cell and laser beam size, but a typical result is shown in Fig. 2. The individual points on this plot correspond to different atomic densities. These data cannot be fit by existing theoretical considerations for this system assuming a constant decay rate $\gamma_{0}$ [15]. This fact is demonstrated by extrapolating the low density end of the curve (where $I_{\text {out }} / I_{\text {in }}$ is nearly 1 ) with a constant decay rate, as shown in the dashed curve and inset. However, taking into account the effect of radiation trapping we can understand this data quite well.

We first analyze these data in terms of a simple theoretical model and then by a detailed numerical simulation. To simplify the analysis we neglect the process of optical pumping and assume a closed system. We include a dephasing of the ground-state coherence with rate $\gamma_{0}$ and neglect population exchange between the ground states. As in previous treatments, we model the effect of radiation trapping by introducing an incoherent pumping rate $R$ from ground states $\left|b_{ \pm}\right\rangle$to excited state $|a\rangle$. $R$ is not a constant, but a function of all parameters of the system [12]. In particular, $R$ depends on the excited-state population, because as 
more population is transferred to the excited state, more radiation will be produced that can eventually cause this incoherent excitation. We assume that $R$ may be of the same order of magnitude as $\gamma_{0}$ but that it is much less then the radiative decay rate $\gamma_{r}$ of transitions $|a\rangle \rightarrow\left|b_{ \pm}\right\rangle$.

We can understand the origin of this incoherent pumping by considering a two-level system coupled to a radiation reservoir. The reduced density matrix operator derived in Weisskopf-Wigner approximation [16 has the form

$$
\begin{aligned}
\dot{\rho}(t)= & -\bar{n}_{t h} \gamma_{r}\left[\hat{\sigma}_{-} \hat{\sigma}_{+} \rho(t)-\hat{\sigma}_{+} \rho(t) \hat{\sigma}_{-}\right]- \\
& \left(\bar{n}_{t h}+1\right) \gamma_{r}\left[\hat{\sigma}_{+} \hat{\sigma}_{-} \rho(t)-\hat{\sigma}_{-} \rho(t) \hat{\sigma}_{+}\right]+\text {H.c. },
\end{aligned}
$$

where $\bar{n}_{t h}$ is thermal average photon number in the reservoir, $\gamma_{r}$ is the atomic decay rate of the upper level, $\hat{\sigma}_{-}=|b\rangle\langle a|$ and $\hat{\sigma}_{+}=|a\rangle\langle b|$. To model atomic excitation by the incoherent radiation in the reservoir, the incoherent pumping rate can be written as $R=2 \gamma_{r} \bar{n}_{t h}$. From Eq. (11) we find the equations of motion for the excited state population:

$$
\dot{\rho}_{a a}=-2 \gamma_{r}\left(\bar{n}_{t h}+1\right) \rho_{a a}+2 \gamma_{r} \bar{n}_{t h} \rho_{b b} .
$$

In an optically thin atomic medium the probability of photon reabsorption is small and $\bar{n}_{t h}=0$. However, in optically thick media photons diffuse slowly and $\bar{n}_{t h} \neq 0$. The value of $\bar{n}_{t h}$ can be estimated from a rate equation

$$
\dot{\bar{n}}_{t h}=-r_{e} \bar{n}_{t h}+r_{a} \rho_{a a},
$$

where $r_{e}$ is the photon escape rate and $r_{a}$ is the pumping rate due to the atomic decay. Both $r_{e}$ and $r_{a}$ depend on the geometry of the system and on the atomic density. In steady state, $\bar{n}_{t h}=r_{a} \rho_{a a} / r_{e}$, and it follows from Eq. (2) and $\rho_{a a}+\rho_{b b}=1$ that $r_{e}>r_{a}$.

It is convenient to formally introduce a function $f(N)$ defined by $r_{a} / r_{e}=f /(1+f)$ which characterizes the radiation trapping, such that $f(N) \geq 0$ and $f(N=0)=0$. In the case when most population is in the ground state, $\rho_{a a} \ll 1$, we see from Eq. (2) that $\dot{\rho}_{a a} \approx-2 \gamma_{r} \rho_{a a} /(1+f)$. In the limit of low light intensity $|\Omega| \ll \gamma_{r}$ and weak radiation 
trapping $\bar{n}_{t h} \ll 1$ the cw light propagation obeys $d|\Omega|^{2} / d z \simeq-2 \kappa \gamma_{r} \rho_{a a}$, where $\Omega$ is the Rabi frequency of the transition, $\kappa=(3 / 8 \pi) N \lambda^{2} \gamma_{r}, \lambda$ is the wavelength, and $z$ is the distance of propagation through the medium. We thus have a simple equation for the incoherent pumping rate due to radiation trapping:

$$
R=-\frac{1}{\kappa} \frac{f(N)}{1+f(N)} \frac{\mathrm{d}}{\mathrm{d} z}|\Omega|^{2}
$$

This is a very intuitively appealing model: radiation trapping can exist only if the coherent radiation is absorbed by the system and is scattered due to spontaneous emission.

We now return to the problem of radiation trapping in a three-level system with EIT. Because the Doppler distribution depends on atomic density (temperature), it is simplest to study radiation trapping in the Doppler-free limit of EIT, i.e., when the absorption and the dispersion do not depend on the width of the Doppler distribution $W_{d}$. Doppler averaging shows that this condition is fulfilled for relatively large light intensities $|\Omega(z)| \gg W_{d} \sqrt{\gamma_{0} / \gamma_{r}}$ for any $z$.

The stationary propagation of the right and left circular polarized electric field components through the atomic vapor is described by Maxwell-Bloch equations in the slowlyvarying amplitude and phase approximation [16]. We solve the equations by considering only the lowest order in $\gamma_{0}, R$ and $\delta_{0}$, assuming $\left|\Omega_{-}(z)\right|^{2} \approx\left|\Omega_{+}(z)\right|^{2}$, where $\Omega_{ \pm}$are the complex Rabi-frequencies of the two optical fields. We separately consider the spatial evolution of the amplitudes and phases of these complex Rabi-frequencies by writing $\Omega_{ \pm}(z)=\left|\Omega_{ \pm}(z)\right| \mathrm{e}^{\mathrm{i} \phi_{ \pm}(\mathrm{z})}$, and derive equations for the total intensity $|\Omega|^{2}$ and the relative phase $\phi=\phi_{-}-\phi_{+}$

$$
\begin{aligned}
\frac{\mathrm{d}}{\mathrm{d} z}|\Omega|^{2} & =-\kappa\left(\gamma_{0}+R\right), \\
\frac{\mathrm{d}}{\mathrm{d} z} \phi & =\delta_{0} \frac{\kappa}{|\Omega|^{2}} .
\end{aligned}
$$

To solve these equations we must specify the functional form of the incoherent pumping rate $R$ in the three level configuration. From the general properties of radiation trapping [9] and from the results from radiation trapping in a two-level system, we assume that in the case of Doppler-free EIT the incoherent pumping can be modeled by Eq. (4) as in the case of a 
two-level system. As we shall see, this model works very well.

With this form for $R$, Eq. (5) can be easily solved and we arrive at

$$
\left|\frac{\Omega(z)}{\Omega(0)}\right|^{2}=1-\frac{\gamma_{0} \kappa z}{|\Omega(0)|^{2}}(1+f(N))
$$

so from Eq. (雨) we have $R=f(N) \gamma_{0}$. Integration of Eq. (6) for the phase yields

$$
\left.\frac{d \phi(z)}{d B}\right|_{B \rightarrow 0}=\frac{2 \mu_{B}}{\hbar\left(\gamma_{0}+R\right)} \ln \left|\frac{\Omega(0)}{\Omega(z)}\right|^{2} .
$$

Detection of $|\Omega(L) / \Omega(0)|^{2}$ and $d \phi(L) / d B$, allows us to infer the value of the coherence decay rate as a function of the atomic density and estimate the radiation trapping effect. Thus we see that for optically thick media the coherence decay rate increases with the density.

For smaller intensities $|\Omega(z)| \ll W_{d} \sqrt{\gamma_{0} / \gamma_{r}}$, Doppler-free EIT is not established, so the approximation Eq. (4) is not valid and we do not discuss this regime here.

Based on the low-density data in Fig. 2 (for which radiation trapping is negligible) Eqs.(17) and (8) allow us to determine the coherence decay rate to be $\gamma_{0} \approx 0.004 \gamma_{r}$. Given this value, we can then use the high density set of these data and Eq. (8) to obtain the incoherent pumping rate $R$ due to radiation trapping. The dependence is shown by the dots in Fig. 3. In general, the functional form of $R$ is not an "absolute" and it changes if the cell geometry or laser beam size changes, which is a key signature of the effect of radiation trapping.

The probability of photon reabsorption becomes significant when the medium becomes optically thick on the length scale of the atomic cell size [9] (under the Doppler-free EIT condition almost all atomic population is in the ground state), or

$$
\frac{3}{8 \pi} N \lambda^{2} d \frac{\gamma_{r}}{W_{d}}>1
$$

For our experiment $\gamma_{r} / \Delta_{D} \approx 0.01$, so Eq. (9) is fulfilled for $N>5 \times 10^{10} \mathrm{~cm}^{-3}$. For densities less than this, radiation trapping is negligible and we have $R \approx 0$. Above this value there are two distinct regimes of behavior, with both seen in Fig. 3. At low density we have that $R$ increases linearly with density due to photon absorption and emission within 
the cell. If the atomic beam is narrower than the radius of the atomic cell, as we have in our experiment $(d \sim 0.1 D)$, the next regime occurs for densities when photon reabsorption becomes significant inside the laser beam. In our case this is $N>5 \times 10^{11} \mathrm{~cm}^{-3}$.

To confirm our simple analytical calculations we have also made detailed numerical simulations of the experiment. We have considered light propagation in a thirteen-level Dopplerbroadened system corresponding to the $F=2 \rightarrow F^{\prime}=1,2$ transition in ${ }^{87} \mathrm{Rb}$. The decay of the atomic coherence was modeled by finite time of the flight through the laser beam (an open system). We solved the density matrix equations in steady state using the coherence decay rate as a fit parameter. In other words, we choose the effective coherence decay rate $\gamma_{0}+R$ in such a way that our numerical points for the dispersion $d \phi / d B$ and intensity $I_{\text {out }} / I_{\text {in }}$ corresponds to the experimental results. This is shown in the solid line in Fig. 2. The dependence for $R / \gamma_{0}$ obtained this way is shown in the solid line in Fig. 3. We see that the simple analytical analysis of the data coincides with the simulations for low atomic densities and diverges slightly for high densities. We explain this difference by inadequate intensity of the laser light. The maximum intensity of our laser $\left(\sim 100 \mathrm{~mW} / \mathrm{cm}^{2}\right)$ corresponds to a Rabi frequency $\left|\Omega_{0}\right| \sim 3.6 \gamma_{r}$, which lies on the edge of Doppler-free region determined by $\left|\Omega_{0}\right| \geq W_{d} \sqrt{\gamma_{0} / \gamma_{r}} \approx 6 \gamma_{r}$. The absorption further decreases the intensity resulting in the Doppler broadening becoming important, unlike in our simplified calculations.

Finally, we note that the observations reported here cannot be explained by spin exchange collisions between the atoms. The collisional cross section for Rb atoms is approximately $2 \times 10^{-14} \mathrm{~cm}^{2}$ [10 which results in a coherence decay rate $\gamma_{0} \approx 2 \times 10^{-5} \gamma_{r}$ for the densities reported here. This is approximately two orders of magnitude less than the time-of-flight limited coherence decay rate $\gamma_{0} \approx 4 \times 10^{-3} \gamma_{r}$ that we measured.

In conclusion, we have shown both experimentally and theoretically that the effect of radiation trapping enhances the decay rate of the atomic coherence established by linearly polarized laser radiation between Zeeman sublevels. This effect leads to significant increase of the residual absorption in EIT experiments with optically thick atomic vapors.

The authors gratefully acknowledge useful discussions with D. Budker, M. Fleischhauer, 
L. Holberg, E. E. Mikhailov, Y. V. Rostovtsev, V. A. Sautenkov, V. L. Velichansky, R. Wynands, and V. Yashchuk, and the support from the Office of Naval Research, the National Science Foundation, and the Welch Foundation. 


\section{REFERENCES}

[1] E. Arimondo, G. Orriols, Nuovo Cimento Lett. 17, 333 (1976); H. R. Gray, R. M. Whitley, C. R. Stroud, Opt. Lett. 3, 218 (1978); H. I. Yoo, J. H. Eberly, Phys. Reports 118, 239 (1985).

[2] E. Arimondo, in Progress in Optics edited by E. Wolf, Vol. XXXV, p.257 (Elsevier Science, Amsterdam, 1996).

[3] For reviews on EIT see S. E. Harris, Phys. Today p. 36, June (1997); J. P. Marangos, J. Mod. Opt. 45, 471 (1998).

[4] A. S. Zibrov et al., Phys. Rev. Lett. 75, 1499 (1995); G. G. Padmabandu et al., ibid., 76, 2053 (1996). J. Mompart and R. Corbalan, Quantum Semiclass. Opt. 2, R7 (2000).

[5] M. O. Scully, Phys. Rep. 219, 191 (1992).

[6] S. E. Harris, J. E. Field, and A. Imamoglu, Phys. Rev. Lett. 64, 1107 (1990); K. Hakuta, L. Marmet, and B. P. Stoicheff, ibid., 66, 596 (1991); P. Hemmer et al., Opt. Lett. 20, 982 (1995); M. Jain et al., Phys. Rev. Lett. 77, 4326 (1996); A. S. Zibrov, M. D. Lukin, and M. O. Scully, ibid., 83, 4049 (1999); A. V. Sokolov et al., ibid., 85, 562 (2000).

[7] M. O. Scully, Phys. Rev. Lett. 67, 1855 (1991); A. S. Zibrov et al., ibid., 76, 3935 (1996).

[8] M. Fleischhauer, M. O. Scully, Phys. Rev. A 49, 1973 (1994); H. Lee, M. Fleischhauer, M. O. Scully, ibid., 58, 2587 (1998).

[9] A. F. Molisch, B. P. Oehry, Radiation Trapping in Atomic Vapours (Clarendon Press, Oxford), 1998.

[10] W. Happer, Rev. Mod. Phys. 44, 169 (1972).

[11] D. Peterson, L. W. Anderson, Phys. Rev. A 43, 4883 (1991); T. Scholz et al., ibid., 53, $2169(1996)$. 
[12] G. Ankerhold et al., Phys. Rev. A 48, R4031 (1993); M. Fleischhauer Europhys. Lett. 45, 659 (1999).

[13] W. Gawlik, in Modern Nonlinear Optics edited by M. Evans and S. Kielich, Vol. LXXXV of Advances in Chemical Physics Series (Wiley, New York, 1994), part 3, p. 733; D. Budker, D. J. Orlando, V. Yashchuk, Am. J. Phys. 67, 589 (1999); V. A. Sautenkov et al., Phys. Rev. A 62, 023810 (2000); F. Schuller, D. N. Stacey, ibid., 60, 973 (2000).

[14] D. Budker et al., Phys. Rev. A 62, 043403 (2000).

[15] M. Fleischhauer, A. B. Matsko and M. O. Scully, Phys. Rev. A 62, 013808 (2000).

[16] M. O. Scully and M. S. Zubairy, Quantum Optics (Cambridge University Press, Cambridge) 1996, p. 252. 


\section{FIGURES}

FIG. 1. Diagram showing the experimental setup; inset: Idealized three-level $\Lambda$-scheme considered in the theoretical calculations.

FIG. 2. The dependence of rotation rate $d \phi / d B$ on transmission through the system $I_{\text {out }} / I_{\text {in }}$ : experimental (dots), previous theory with $\gamma_{0}=0.004 \gamma_{r}$ (dashed line), and obtained by numerical simulation including radiation trapping. (solid line).

FIG. 3. The incoherent pumping rate $R / \gamma_{0}$ due to radiation trapping as a function of atomic

density $N$ : calculated by applying Eq. (8) to the data (dots) and obtained by numerical simulation (solid line). 


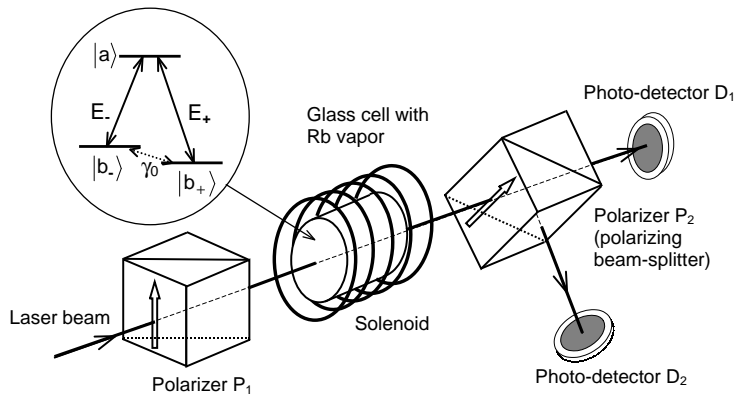

Radiation trapping in coherent media Matsko et al. - Fig. 1 


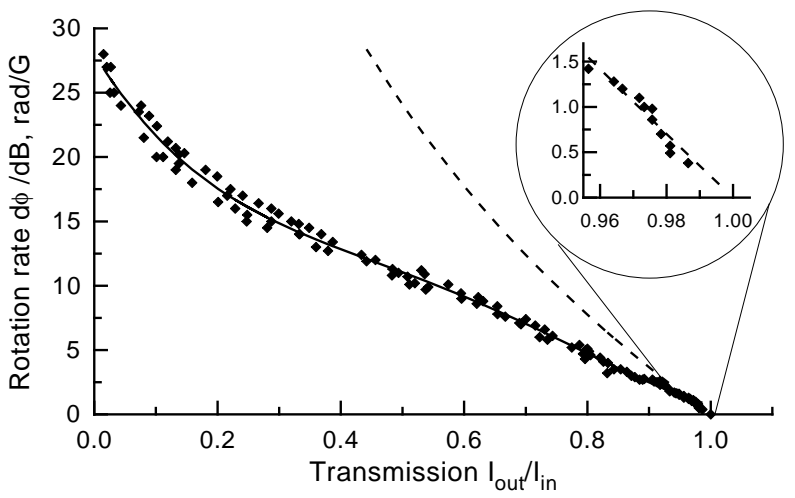

Radiation trapping in coherent media

Matsko et al. - Fig. 2 


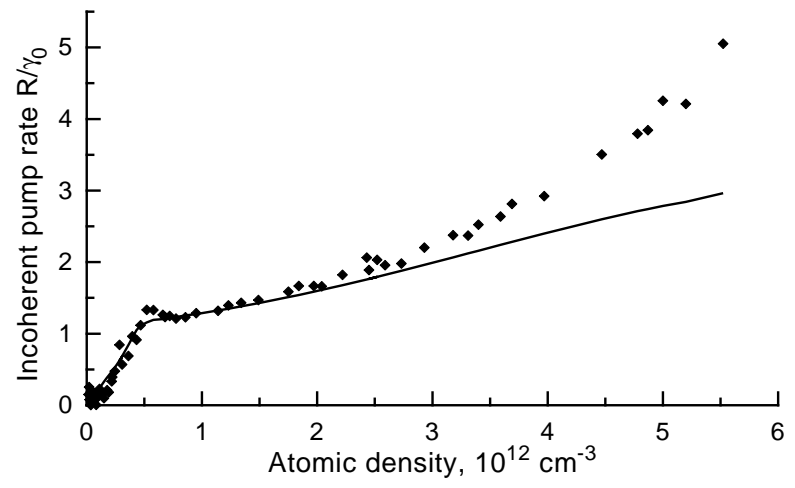

Radiation trapping in coherent media Matsko et al. - Fig. 3 